\title{
Predictors of Posttraumatic Growth Following Bone Marrow Transplantation for Cancer
}

\author{
Michelle R. Widows, Paul B. Jacobsen, Margaret Booth-Jones, and Karen K. Fields \\ University of South Florida
}

\begin{abstract}
There is growing recognition that the experience of cancer can have a positive as well as a negative psychological impact. This longitudinal study sought to identify predictors of posttraumatic growth among cancer patients $(N=72)$ undergoing bone marrow transplantation. Greater posttraumatic growth in the posttransplant period was related to younger age; less education; greater use of positive reinterpretation, problem solving, and seeking alternative rewards as coping strategies in the pretransplant period; more stressful appraisal of aspects of the transplant experience; and more negatively biased recall of pretransplant levels of psychological distress. Findings partially support J. A. Schaefer and R. H. Moos's (1992) model of life crises and personal growth and also suggest that temporal self-comparisons contribute to the experience of posttraumatic growth
\end{abstract}

Keywords: posttraumatic growth, cancer, bone marrow transplantation, temporal comparison

The diagnosis and treatment of cancer can be a traumatic experience with long-lasting psychological effects. Consistent with this view, a number of studies have documented the presence of posttraumatic stress disorder (PTSD), as defined in the Diagnostic and Statistical Manual of Mental Disorders (4th ed.; DSM-IV; American Psychiatric Association, 1994), in response to cancer diagnosis and treatment (Alter et al., 1996; Andrykowski, Cordova, Studts, \& Miller, 1998; Green et al., 1998; Widows, Jacobsen, \& Fields, 2000). A growing body of research supports the possibility that cancer diagnosis and treatment might also result in positive psychological outcomes or benefits. Qualitative and descriptive studies suggest that some patients experience greater appreciation of life and changed priorities, greater satisfaction with self, better relationships with family and others, and positive changes in religious beliefs and spirituality as a result of their experience with cancer (Belec, 1992; Curbow, Legro, Baker, Wingard, \& Somerfield, 1993; Fromm, Andrykowski, \& Hunt, 1996; Zemore, Rinholm, Shepel, \& Richards, 1989; Zemore \& Shepel, 1989).

Individual differences in reports of positive changes and benefits have been found to be associated with a number of demographic and clinical variables, with higher levels of positive changes and benefits related to the female gender (Curbow, Legro, et al., 1993; Tempelaar et al., 1989), younger age (Curbow, Legro, et al., 1993; Klauer, Ferring, \& Filipp, 1998; Lichtman, Taylor, \& Wood, 1987; Tempelaar et al., 1989), married status (Andrykowski, Brady, \& Hunt, 1993; Curbow, Legro, et al., 1993; Rieker, Edbril, \& Garnick, 1985), higher income (Carpenter,

Michelle R. Widows and Paul B. Jacobsen, Department of Psychology, University of South Florida; Margaret Booth-Jones and Karen K. Fields, Department of Interdisciplinary Oncology, University of South Florida.

Correspondence concerning this article should be addressed to Paul B. Jacobsen, Department of Psychology, PCD4118, University of South Florida, 4202 East Fowler Avenue, Tampa, FL 33620. E-mail: jacobsen@ moffitt.usf.edu
Brockopp, \& Andrykowski, 1999), less invasive surgery (Lichtman et al., 1987), more recent treatment (Fromm et al., 1996; Tempelaar et al., 1989), more intensive chemotherapy regimens (Tempelaar et al., 1989), greater degree of risk with transplant (Fromm et al., 1996), and remitted disease status (Collins, Taylor, \& Skokan, 1990). Psychosocial variables associated with reports of more positive outcomes include fewer role activities (Tempelaar et al., 1989), better sexual functioning (Rieker et al., 1985), greater levels of self-esteem (Carpenter et al., 1999; Tempelaar et al., 1989), greater levels of optimism (Curbow, Somerfield, Baker, Wingard, \& Legro, 1993), and use of a variety of coping strategies rather than one primary coping strategy (Collins et al., 1990).

Although progress has been made in understanding positive outcomes following cancer diagnosis and treatment, the literature has been characterized by several methodological limitations. First and foremost, research in this area has generally relied on unstandardized interview methods to assess positive outcomes. Few studies have used standardized measures of known reliability and validity designed to yield quantitative information about positive outcomes. Second, those studies presenting quantitative information consist largely of descriptions of the relative frequency of different types of positive outcomes. Third, the few studies that have examined variables associated with individual differences in positive outcomes have generally not been guided by theorydriven hypotheses.

One potentially useful way of conceptualizing positive outcomes related to cancer and its treatment is as "posttraumatic growth." Tedeschi and colleagues (Tedeschi \& Calhoun, 1995; Tedeschi, Park, \& Calhoun, 1998) theorized that stressful or traumatic events have the potential to lead to positive outcomes (growth) as a function of cognitive processing that leads to the questioning of one's assumptions about the world and the development of new schemas and coping strategies. To measure this construct, Tedeschi and Calhoun (1996) developed the Posttraumatic Growth Inventory (PTGI). This self-report measure has been used in a number of different populations, including bereaved 
parents (Polatinsky \& Esprey, 2000), parents of pediatric leukemia patients (Best, Streisand, Catania, \& Kazak, 2001), accident and assault victims (Snape, 1997), and violent crime victims (Peltzer, 2000). In the first published study to use this measure in a cancer population, Cordova, Cunningham, Carlson, and Andrykowski (2001) examined the characteristics and correlates of posttraumatic growth in women treated with surgery, chemotherapy, or radiotherapy for nonmetastatic breast cancer. Results indicated that breast cancer patients showed greater posttraumatic growth in terms of relating to others, appreciation of life, and spiritual change than did age-matched controls (who completed the PTGI by reporting on changes experienced over an interval equal to the time since cancer diagnosis for their matched breast cancer counterparts). Cross-sectional analyses revealed that greater posttraumatic growth among breast cancer patients was associated with greater perceived life threat, more talking about breast cancer, higher income, and longer time since diagnosis.

The current study had four objectives. One aim was to extend the research by Cordova et al. (2001) on posttraumatic growth by focusing on bone marrow transplantation (BMT) recipients, a population of cancer patients undergoing more aggressive treatment and at greater risk for mortality than are women with nonmetastatic breast cancer. A second aim was to identify psychosocial predictors of individual differences in posttraumatic growth by using a longitudinal design. On the basis of Schaefer and Moos's (1992) theory of life crises and personal growth and prior research on psychological adjustment in cancer patients (Brady \& Helgeson, 1999; Lichtman et al., 1987; Osowiecki \& Compas, 1999; Zemore \& Shepel, 1989), we hypothesized that greater levels of posttraumatic growth following BMT would be predicted by greater use of approach-based coping strategies and greater perceived social support prior to transplant. On the basis of the view that higher levels of stress should stimulate greater posttraumatic growth (Calhoun \& Tedeschi, 1998), we also hypothesized that patients who were more distressed prior to transplant and appraised the transplant period as more stressful would report greater posttraumatic growth. A third aim was to explore the relationship of posttraumatic growth to posttransplant levels of psychological distress and PTSD symptomatology. As previous research has yielded mixed findings regarding the concurrent relationship between positive outcomes and mental health in cancer patients (Cordova et al., 2001; Curbow, Somerfield, et al., 1993; Fromm et al., 1996; Katz, Flasher, Cacciapaglia, \& Nelson, 2001), no specific hypotheses were offered. In addition, the current study sought to explore whether posttraumatic growth was differentially related to actual versus perceived changes in psychological distress over time. This last aim was guided by temporal comparison theory (Albert, 1977) and research suggesting that certain aspects of perceived growth are illusory and attributable to negatively biased recall (i.e., derogation) of past attributes or performance (McFarland \& Alvaro, 2000; Wilson \& Ross, 2001).

\section{Method}

\section{Participants}

Participants were patients who had completed BMT for cancer at the $\mathrm{H}$. Lee Moffitt Cancer Center (HLMCC). To be eligible for study participation, these patients also had to (a) be greater than 18 years of age, (b) be able to speak and read English, (c) have a sixth-grade education, (d) have no clinical evidence of disease at last medical follow-up, (e) be at least 6 months postdischarge at time of follow-up assessment, and (f) have completed a routine clinical psychosocial evaluation prior to transplant. Approximately $95 \%$ of patients undergoing BMT at HLMCC during the period of this study completed this evaluation.

Of 114 BMT recipients meeting these eligibility criteria, 19 (17\%) could not be reached, $1(1 \%)$ declined participation, and $22(19 \%)$ completed the pre-BMT psychosocial evaluation but did not return the post-BMT questionnaire packet. Thus, complete data were obtained from a final sample of 72 BMT recipients $(63 \%)$.

\section{Procedure}

Potential study participants who had completed BMT at least 6 months prior to follow-up data collection were identified with the HLMCC registry. Study eligibility was determined through registry review and consultation with HLMCC Blood and Marrow Transplantation Program team members. BMT recipients meeting eligibility requirements were initially contacted via an introductory letter from the director of the HLMCC Blood and Marrow Transplantation Program. Individuals were then contacted by telephone to obtain verbal consent for study follow-up participation. BMT recipients who verbally agreed to participate were mailed an informed consent form, questionnaire packet, and stamped envelope in which to return the study materials. A research team member telephoned participants 1 week following the initial packet mailing to answer any questions and provide a reminder to promptly return the study materials. A second telephone reminder occurred if study materials had not been received within 2 weeks.

\section{Pre-BMT Measures}

Coping strategies. The Coping Responses Inventory (Moos, 1993) is a 48-item self-report measure of strategies that individuals use in dealing with stressful events. Responses to these items are used to compute scores for scales that have been labeled Logical Analysis, Positive Reappraisal, Seeking Guidance and Support, Problem Solving, Cognitive Avoidance, Acceptance or Resignation, Seeking Alternative Rewards, and Emotional Discharge. The first four scales are conceptualized as measuring approach coping, and the latter four scales are conceptualized as measuring avoidance coping (Moos, 1993). Respondents rated on a 4-point scale ranging from 0 (not at all) to 3 (fairly often) how often in the past month they had used each strategy to deal with their cancer and its treatment. Internal consistency $(\alpha)$ for Coping Responses Inventory subscale scores ranged from .51 (Problem Solving) to .74 (Seeking Alternative Rewards) in the current study. A total approach coping score was created by summing the four approach coping subscale scores $(\alpha=.88)$, and a total avoidance coping score was created by summing the four avoidance coping subscale scores $(\alpha=.79)$.

Social support. The Interpersonal Support Evaluation List-Short Form (ISEL-SF; Peirce, Frone, Russell, \& Cooper, 1996) is a 15-item self-report measure derived from the 40-item Interpersonal Support Evaluation List (Cohen, Mermelstein, Kamarck, \& Hoberman, 1985). The ISEL-SF retains five items from each of the three subscales from the original Interpersonal Support Evaluation List (Tangible Support, Appraisal Support, and Belonging Support). Respondents indicated how true or false each statement was for them by using a 4-point Likert scale ranging from 1 (completely false) to 4 (completely true). Internal consistency of the total ISEL-SF scale score was .83.

Psychological distress. The Profile of Mood States-Short Version (POMS-SV; Shacham, 1983) is a 37-item self-report measure of current affective state derived from the 65-item Profile of Mood States (McNair, Lorr, \& Droppleman, 1992). Respondents indicated the degree to which they experienced each item within the previous week on a 5-point scale 
ranging from 0 (not at all) to 4 (extremely). The POMS-SV retains all of the original Profile of Mood States subscales: Tension-Anxiety, Depression-Dejection, Anger-Hostility, Vigor, Fatigue, and ConfusionBewilderment. A total mood disturbance (TMD) score was calculated by summing the subscale scores, with the Vigor scale reverse coded. Internal consistency of the pre-BMT TMD score was .94.

\section{Post-BMT Measures}

Disease and treatment data. The HLMCC computerized patient database and patient medical charts were reviewed at the time of the follow-up assessment to obtain information regarding disease and treatment characteristics. Variables assessed included cancer diagnosis, clinical rating of risk of disease recurrence or progression at time of transplant, ${ }^{1}$ transplant type (autologous vs. allogeneic), hospitalization status at transplant (inpatient vs. outpatient), length of hospitalization for transplantation, and number of days to engraftment. ${ }^{2}$

Demographic data. Demographic data were obtained through the use of a standard self-report questionnaire. Variables assessed included age, gender, ethnicity, education, income, and marital and employment status.

Posttraumatic growth. The Posttraumatic Growth Inventory (PTGI; Tedeschi \& Calhoun, 1996) is a 21-item self-report measure reflecting the tendency to perceive positive change and benefits following traumatic events. In the present study, respondents rated the degree to which each change occurred as a result of their experience with cancer and its treatment on a 6-point scale ranging from 0 (I did not experience this change) to 5 (I experienced this change to a great degree). The PTGI yields a total score and scores for five factorially derived subscales: New Possibilities, Relating to Others, Personal Strength, Spiritual Change, and Appreciation of Life. Internal consistency of the PTGI total score was .93.

Stress appraisal. The six-item Trauma Experience Questionnaire (Widows et al., 2000) measures patients' subjective appraisals of the stressfulness of BMT. Separate items assess patients' retrospective perceptions of helplessness, hopelessness, loss of control, pain, distress, and concerns about dying during the course of their BMT. Ratings were made on a Likert scale ranging from 1 (none or not at all) to 4 (severe or a lot), with higher scores reflecting more stressful appraisals of the BMT experience. Items are similar to those used by Neumann (1991) to study posttraumatic effects in myocardial infarction patients. Internal consistency of the Trauma Experience Questionnaire total score was .77.

PTSD symptomatology. The PTSD Checklist-Civilian Version (PCL-C; Weathers, Litz, Herman, Huska, \& Keane, 1993) is a 17-item self-report measure that comprises items reflecting PTSD symptom criteria according to the $D S M-I V$. In the present study, participants were instructed to respond to items in terms of their experience with cancer and its treatment and to rate each item in terms of how much it had bothered them in the past month on a scale ranging from 1 (not at all) to 5 (extremely). The PCL-C yields a total score as well as subscale scores for Intrusions, Avoidance/Numbing, and Arousal. Internal consistency of the PCL-C total scale score was .91 .

Psychological distress. The POMS-SV was readministered at the post-BMT assessment to measure current psychological distress. Internal consistency of the post-BMT TMD score was .97. Post-BMT TMD scores were used with pre-BMT TMD scores to calculate actual change in psychological distress (pre-BMT TMD minus post-BMT TMD).

Recall of psychological distress. BMT recipients completed the POMS-SV a second time during the post-BMT assessment by using instructions designed to yield a renewed judgment of their psychological distress prior to transplant. Specifically, participants were instructed to rate each item for "how you were feeling when you completed the psychosocial questionnaires before your BMT." Internal consistency of the recalled pre-BMT TMD score was 97 . Recalled pre-BMT scores were used to calculate perceived change in psychological distress (post-BMT TMD minus recalled pre-BMT TMD) and biased recall of pretransplant psychological distress (recalled pre-BMT TMD minus pre-BMT TMD).

\section{Statistical Analyses}

Bivariate correlations of demographic, clinical, and psychosocial variables with posttraumatic growth were analyzed by using Pearson productmoment correlations or Spearman rank correlations as appropriate. Differences between participants' ratings of their post-BMT, pre-BMT, and recalled pre-BMT psychological distress were analyzed by using pairedsample $t$ tests. Finally, hierarchical multiple regression analysis was used to identify the relative contribution of the psychosocial variables of interest to posttraumatic growth. The three psychosocial variables that were measured pre-BMT and were hypothesized to be related to posttraumatic growth (i.e., social support, psychological distress, and approach coping) were entered into the equation on the first step, followed by the one psychosocial variable that was measured post-BMT and was also hypothesized to be related to posttraumatic growth (i.e., stress appraisal). Finally, biased recall of pretransplant distress (recalled pre-BMT TMD minus pre-BMT TMD) was entered on the third step to explore whether it accounted for additional significant variability in posttraumatic growth beyond that accounted for by variables hypothesized to be related to posttraumatic growth. Statistical significance for all analyses was set at $p \leq .05$.

\section{Results}

\section{Participant Characteristics}

Participants ranged from 25 to 66 years of age $(M=47.62$, $S D=10.04)$ and were an average of 24.05 months $(S D=10.01$; range $=8-47$ ) post-BMT at the time of follow-up questionnaire completion. BMT recipients treated as inpatients $(n=63)$ were hospitalized for an average of 23.37 days $(S D=10.49$; range $=$ 13-77) for transplant. The average time to engraftment following transplant was 12.96 days $(S D=4.47$; range $=8-31)$. Other demographic and clinical characteristics of the sample are described in Table 1.

Preliminary analyses of demographic and clinical data were conducted to identify possible differences between the former BMT recipients who completed all study participation requirements $(N=72)$ and those who could not be contacted or did not return follow-up questionnaire packets $(n=42)$. Results of $t$-test, chi-square test, and Fisher's exact test comparisons indicated that study participants and nonparticipants did not differ significantly $(p>.05)$ with regard to gender, diagnosis, prognosis, transplant

\footnotetext{
${ }^{1}$ Patients were classified as being at low, intermediate, or high risk of disease recurrence or progression after BMT on the basis of their disease status at time of transplant. The following diseases were classified as low risk: breast cancer, Stage II; acute myelogenous leukemia, acute lymphoblastic leukemia, lymphomas, other leukemias, CR1-CR3; chronic myelogenous leukemia, CP1; and multiple myeloma, CR. The following diseases were classified as intermediate risk: breast cancer, Stage III; acute myelogenous leukemia, acute lymphoblastic leukemia, lymphomas, other leukemias, REL1 and REL2; chronic myelogenous leukemia, AP1 and $\mathrm{CP} 2$; and multiple myeloma, PR and MR. The following diseases were classified as high risk: breast cancer, Stage IV and metastatic; acute myelogenous leukemia, acute lymphoblastic leukemia, lymphomas, other leukemias, REL3; chronic myelogenous leukemia, AP2 and BP1; and multiple myeloma, systemic.

${ }^{2}$ Number of days to engraftment represents the interval between the administration of transplanted blood or marrow cells to the patient and the production of new white blood cells, red blood cells, and platelets. A longer time to engraftment interval is generally associated with greater treatmentrelated morbidity due primarily to increased risk of bleeding and infection.
} 
Table 1

Demographic and Clinical Characteristics of Study Participants $(N=72)$

\begin{tabular}{|c|c|c|}
\hline Variable & $n$ & $\%$ \\
\hline \multicolumn{3}{|l|}{ Gender } \\
\hline Female & 53 & 74 \\
\hline Male & 19 & 26 \\
\hline \multicolumn{3}{|l|}{ Ethnicity } \\
\hline African American & 5 & 7 \\
\hline Caucasian & 61 & 85 \\
\hline Hispanic & 6 & 8 \\
\hline \multicolumn{3}{|l|}{ Marital status } \\
\hline Currently married & 55 & 76 \\
\hline Unmarried & 17 & 24 \\
\hline \multicolumn{3}{|l|}{ Education } \\
\hline Some high school & 4 & 6 \\
\hline High school graduate & 13 & 18 \\
\hline Some college or specialized training & 26 & 36 \\
\hline College graduate & 19 & 26 \\
\hline Graduate training or degree & 10 & 14 \\
\hline \multicolumn{3}{|l|}{ Employment status } \\
\hline Full-time & 34 & 47 \\
\hline Part-time & 6 & 8 \\
\hline On leave & 3 & 4 \\
\hline Disabled & 16 & 23 \\
\hline Not employed & 13 & 18 \\
\hline \multicolumn{3}{|l|}{ Annual income } \\
\hline$<\$ 19,999$ & 9 & 13 \\
\hline$\$ 20,000-\$ 39,999$ & 11 & 15 \\
\hline$\$ 40,000-\$ 59,999$ & 20 & 28 \\
\hline$\$ 60,000-\$ 100,000$ & 18 & 25 \\
\hline$>\$ 100,000$ & 8 & 11 \\
\hline Declined to report & 6 & 8 \\
\hline \multicolumn{3}{|l|}{ Diagnosis } \\
\hline Breast cancer & 37 & 51 \\
\hline Multiple myeloma & 9 & 13 \\
\hline Non-Hodgkin’s lymphoma & 9 & 13 \\
\hline Chronic myelogenous leukemia & 7 & 10 \\
\hline Hodgkin's disease & 5 & 7 \\
\hline Acute myelogenous leukemia & 3 & 4 \\
\hline Acute lymphoblastic leukemia & 2 & 3 \\
\hline \multicolumn{3}{|l|}{ Risk of disease recurrence or progression } \\
\hline Low & 28 & 39 \\
\hline Intermediate & 28 & 39 \\
\hline High & 16 & 22 \\
\hline \multicolumn{3}{|l|}{ Transplant type } \\
\hline Autologous & 54 & 75 \\
\hline Allogeneic & 18 & 25 \\
\hline \multicolumn{3}{|l|}{ Hospitalization status } \\
\hline Inpatient & 63 & 88 \\
\hline Outpatient & 9 & 12 \\
\hline
\end{tabular}

type, hospitalization status, time to engraftment, or length of hospitalization. Individuals who did not return follow-up questionnaire packets or could not be contacted were found to be significantly younger, $t(113)=-2.58, p=.01$, and further out from transplant, $t(113)=2.65, p<.01$, than were individuals who had completed all aspects of study participation.

\section{Characteristics of Posttraumatic Growth}

The average PTGI total score was $64.67(S D=21.30)$. Total scores ranged from a low of 0 to a high of 101 out of a possible range of 0 to 105 . PTGI items endorsed by individual participants at a "moderate degree" or greater $(\geq 3)$ were tabulated in order to characterize the most and least common forms of posttraumatic growth. Using this criterion, we found that the average participant endorsed 14.70 of the 21 PTGI items $(S D=5.23$; range $=0-21$ ). The five most frequently endorsed items were "An appreciation for the value of my own life" (92\%), "My priorities about what is important in life" (90\%), "Appreciating each day" (90\%), "I learned a great deal about how wonderful people are" (86\%), and "Knowing I can count on people in times of trouble" (85\%). Two items were endorsed by less than $50 \%$ of participants: "New opportunities are available which would not have been otherwise" (32\%) and "I developed new interests" (44\%).

\section{Relation of Demographic and Clinical Variables to Posttraumatic Growth}

Although no hypotheses were proposed, analyses were conducted to examine relationships between demographic variables and posttraumatic growth. As shown in Table 2, there were significant relationships between posttraumatic growth and both age $(p=.05)$ and education $(p=.001)$. Greater posttraumatic growth was more likely to be reported by younger and less well-educated BMT recipients. Posttraumatic growth was not significantly related to gender, ethnicity, marital status, employment status, or annual income ( $p \mathrm{~s}>.05)$.

Table 2 also presents the results of analyses of relationships between clinical variables and posttraumatic growth. Contrary to predictions, posttraumatic growth was unrelated to risk of disease recurrence or progression $(p=.19)$, a variable that may serve as an objective measure of degree of life threat. Exploratory analyses of other clinical variables (cancer diagnosis, hospitalization status, allogeneic vs. autologous transplant, time to engraftment, time since transplantation, and length of hospitalization) also yielded nonsignificant relationships with posttraumatic growth $(p>.05)$

Table 2

Correlations Between Posttraumatic Growth and Demographic and Clinical Variables

\begin{tabular}{lrr}
\hline \multicolumn{1}{c}{ Variable } & \multicolumn{2}{c}{ PTGI total } \\
\cline { 2 - 4 } & $r$ & $p$ \\
\hline Demographic & & \\
Age (years) & -.23 & .05 \\
Gender (female vs. male) & .14 & .26 \\
Ethnicity (Caucasian vs. non-Caucasian) & -.03 & .78 \\
Marital status (married vs. unmarried) & .02 & .89 \\
Education (level) & -.37 & .001 \\
Employment (full-time vs. not full-time) & -.01 & .90 \\
Annual income (\$) & -.13 & .31 \\
Clinical & & \\
Diagnosis (breast vs. other) & -.02 & .90 \\
Recurrence/progression (risk) & .16 & .19 \\
Transplant type (autologous vs. allogeneic) & -.03 & .81 \\
Hospitalization status (inpatient vs. outpatient) & .07 & .56 \\
Time to engraftment (days) & .06 & .60 \\
Length of hospitalization (days) & -.04 & .79 \\
Time since transplant (months) & .12 & .30 \\
\hline
\end{tabular}

Note. $\quad N=72$. PTGI $=$ Posttraumatic Growth Inventory.

${ }^{\text {a }}$ Value reflects Spearman rank correlation statistic. All other values reflect Pearson product-moment correlation statistics. 


\section{Relation of Social Support, Coping, and Appraisal to Posttraumatic Growth}

Analyses were conducted to test hypotheses regarding the relationship of social support, appraisal, and coping to posttraumatic growth (see Table 3). Contrary to predictions, greater social support measured prior to BMT was not related to posttraumatic growth $(p=.67)$. With regard to appraisal, results provided partial support for the hypothesis that greater posttraumatic growth would be associated with more stressful retrospective appraisals of the BMT experience. Although posttraumatic growth was not significantly related to the total stress appraisal score $(p=.09)$, it was significantly positively associated with post-BMT recollections of greater emotional distress $(p=.01)$ and greater concern for life $(p=.02)$ during BMT. With regard to coping, there was partial support for the hypothesis that greater use of approach-based coping strategies prior to BMT would be related to greater posttraumatic growth. Although the total approach coping score was not significantly related to posttraumatic growth $(p=.08)$, greater use of the approach-based strategies of positive reappraisal

Table 3

Correlations Between Posttraumatic Growth and Psychosocial Variables

\begin{tabular}{lll}
\hline \multicolumn{1}{c}{ Variable } & & \\
& \multicolumn{2}{c}{ PTGI total } \\
\cline { 2 - 3 } \multicolumn{1}{c}{ Pre-BMT assessment } & $r$ & $p$ \\
\hline & & \\
& & \\
Interpersonal Support Evaluation List-Short & .05 & .67 \\
Form total score & & \\
Coping Responses Inventory & .21 & .08 \\
Approach coping & .06 & .64 \\
Logical Analysis & .25 & .03 \\
Positive Reappraisal & .11 & .37 \\
Seeking Guidance and Support & .24 & .04 \\
$\quad$ Problem Solving & .28 & .02 \\
Avoidant coping & .16 & .17 \\
Cognitive Avoidance & .14 & .24 \\
Acceptance or Resignation & .23 & .05 \\
Seeking Alternative Rewards & .21 & .08 \\
Emotional Discharge & .03 & .82 \\
Profile of Mood States-Short Version TMD &
\end{tabular}

Post-BMT assessment

\begin{tabular}{lrl} 
Trauma Experience Questionnaire & & \\
Total score & .20 & .09 \\
Pain & .13 & .27 \\
Emotional distress & .31 & .01 \\
Control of life & .01 & .92 \\
Helplessness & -.03 & .83 \\
Hopelessness & .14 & .25 \\
Concern for life & .27 & .02 \\
PTSD Checklist-Civilian Version total score & -.01 & .98 \\
Profile of Mood States-Short Version TMD & -.13 & .28 \\
Differences in psychological distress $^{\text {Biased recall }}{ }^{\mathrm{a}}$ & & \\
Actual change $^{\mathrm{b}}$ & .31 & .01 \\
Perceived change $^{\mathrm{c}}$ & .18 & .14 \\
& .35 & .01 \\
\hline
\end{tabular}

Note. $\quad N=72$. PTGI $=$ Posttraumatic Growth Inventory; BMT $=$ bone marrow transplantation; TMD $=$ total mood disturbance; $\mathrm{PTSD}=$ posttraumatic stress disorder.

${ }^{\mathrm{a}}$ Recalled pre-BMT TMD - pre-BMT TMD. ${ }^{\mathrm{b}}$ Pre-BMT TMD - postBMT TMD. ${ }^{\mathrm{c}}$ Recalled pre-BMT TMD - post-BMT TMD. and problem solving prior to BMT were significantly related to greater posttraumatic growth $(p<.05)$. In the absence of specific hypotheses, exploratory analyses were conducted to examine the relationship between avoidance-based coping measured prior to BMT and posttraumatic growth. Results indicated that the total avoidance coping score was significantly positively related to posttraumatic growth $(p=.02)$. In addition, greater use of the avoidance-based strategy of seeking alternative rewards was significantly related to greater posttraumatic growth $(p=.05)$.

\section{Relation of Psychological Distress and PTSD Symptomatology to Posttraumatic Growth}

Analyses were conducted to test the hypothesis that greater psychological distress in the pretransplant period would predict greater posttraumatic growth. As shown in Table 3, results did not support this hypothesis $(p=.82)$. In addition, results of exploratory analyses (see Table 3 ) indicated that posttraumatic growth was not significantly related to posttransplant levels of psychological distress or PTSD symptomatology $(p>.28)$.

To calculate actual and perceived changes in psychological distress, we compared post-BMT TMD scores with pre-BMT TMD scores and recalled pre-BMT TMD scores. As shown in Table 4, comparison of pre-BMT TMD scores with post-BMT TMD scores indicated that actual change in psychological distress over time was not significant $(p=.72$ ). However, post-BMT TMD scores were significantly lower than recalled pre-BMT TMD scores $(p<.01) .^{3}$ In other words, patients' ratings of posttransplant distress were lower relative to their retrospective ratings of pretransplant distress but not to their actual ratings of pretransplant distress. To calculate the direction and extent of biased recall of psychological distress, we compared recalled pre-BMT TMD scores with pre-BMT TMD scores. As shown in Table 4, recalled pre-BMT TMD scores were significantly greater than pre-BMT TMD scores $(p=.001){ }^{4}$

As shown in Table 3, biased recall of pre-BMT psychological distress (recalled pre-BMT TMD minus pre-BMT TMD) was significantly related to posttraumatic growth $(p=.01) .^{5}$ The direction of the relationship indicates that more negatively biased recall of psychological distress was related to greater posttraumatic growth. Perceived change in psychological distress (recalled preBMT TMD minus post-BMT TMD) was also related to posttraumatic growth, with greater perceived decreases in distress related

\footnotetext{
${ }^{3}$ In light of this significant comparison, additional paired-samples $t$ tests were conducted by using POMS-SV subscales. Post-BMT scores were significantly lower than recalled pre-BMT scores on the Tension-Anxiety, Depression-Dejection, and Confusion-Bewilderment subscales ( $p s \leq .01)$ and significantly higher than recalled pre-BMT scores on the Vigor subscale $(p<.001)$.

${ }^{4}$ In light of this significant comparison, additional paired-sample $t$ tests were conducted by using POMS-SV subscales. Recalled pre-BMT scores were significantly higher than pre-BMT scores on the Tension-Anxiety, Depression-Dejection, Fatigue, and Confusion-Bewilderment subscales $(p s \leq .004)$ and significantly lower than pre-BMT scores on the Vigor subscale $(p<.001)$.

${ }^{5}$ In light of this significant correlation, additional correlational analyses were conducted by using PTGI subscales. More negatively biased recall of psychological distress was significantly related to higher scores on the New Possibilities, Relating to Others, Spiritual Change, and Appreciation of Life subscales of the PTGI ( $p$ s $\leq .05)$.
} 
to greater posttraumatic growth $(p=.01){ }^{6}$ In contrast, actual change in psychological distress from pre- to posttransplant (preBMT TMD minus post-BMT TMD) was unrelated to posttraumatic growth $(p=.14)$.

\section{Multiple Regression Analysis of Variables Related to Posttraumatic Growth}

Hierarchical multiple regression analysis was performed by using the approach described previously to identify the relative contribution of the psychosocial variables of interest to posttraumatic growth. As shown in Table 5, social support, psychological distress, and approach coping measured prior to BMT accounted for $4 \%$ of the variance in posttraumatic growth on the first step of the equation $(p=.39)$. Retrospective appraisals of the stressfulness of the BMT experience accounted for an additional $4 \%$ of the variance in posttraumatic growth on the second step of the equation $(p=.10)$. Finally, biased recall of pretransplant distress accounted for an additional $7 \%$ of the variance in posttraumatic growth on the third step of the equation $(p=.03)$.

\section{Discussion}

Reports of posttraumatic growth were common among these $\mathrm{BMT}$ recipients. Using a rating of moderate degree or better as a criterion, we found that BMT recipients endorsed an average of 14.7 of 21 PTGI items. Comparison with prior research does not suggest that they were more likely to experience posttraumatic growth than were other populations of cancer patients. For example, the average PTGI total score in the present study of 64.7 is very similar to the average scores of 64.1 reported for women with breast cancer who were also assessed an average of 24 months posttreatment (Cordova et al., 2001) and 60.2 reported for women with breast cancer who were assessed an average of 35 months posttreatment (Weiss, 2002).

In general, demographic and clinical variables were unrelated to individual differences in posttraumatic growth. Age and education were exceptions to this pattern, with younger patients and less well-educated patients reporting greater posttraumatic growth. Prior research using the PTGI with cancer patients (Cordova et al., 2001; Sears, Stanton, \& Danoff-Burg, 2003) did not find either of these variables to be related to posttraumatic growth. A number of studies of cancer patients have examined the relationship of age

Table 4

Comparison of Profile of Mood States-Short Version (POMS-SV) Pre-BMT, Post-BMT, and Recall Assessments

\begin{tabular}{lcccc}
\hline $\begin{array}{c}\text { POMS-SV total mood } \\
\text { disturbance }\end{array}$ & $M$ & $S D$ & $t$ & $p$ \\
\hline Pre-BMT & 12.08 & 17.26 & -0.36 & .72 \\
Post-BMT & 13.31 & 27.61 & & \\
Recalled pre-BMT & 26.19 & 30.51 & 4.56 & .001 \\
Pre-BMT & 12.08 & 17.26 & & \\
& & & & \\
Recalled pre-BMT & 26.19 & 30.51 & 3.18 & .01 \\
Post-BMT & 13.31 & 27.61 & & \\
\hline
\end{tabular}

Note. $\quad N=72 . \mathrm{BMT}=$ bone marrow transplantation.
Table 5

Multiple Regression Analyses of Posttraumatic Growth

\begin{tabular}{lrccc}
\hline \multicolumn{1}{c}{ Variable } & $\beta$ & $\Delta R^{2}$ & Cumulative $R^{2}$ & $p^{\mathrm{a}}$ \\
\hline Step 1 & & .04 & .04 & .39 \\
$\quad$ Social support (ISEL-SF) & -.04 & & & \\
$\quad \begin{array}{l}\text { Psychological distress } \\
\quad \text { (pre-BMT TMD) }\end{array}$ & .06 & & & \\
$\quad$ Approach coping (CRI) & .22 & & & \\
Step 2 & .22 & .04 & .08 & .10 \\
$\quad$ Stress appraisal (TEQ) & .07 & .15 & .03 \\
Step 3 & .31 & & & \\
$\quad$ Biased recall & & & &
\end{tabular}

Note. $\quad N=72$. ISEL-SF $=$ Interpersonal Support Evaluation List-Short Form; $\mathrm{BMT}=$ bone marrow transplantation; TMD $=$ total mood disturbance; CRI $=$ Coping Responses Inventory; TEQ $=$ Trauma Experience Questionnaire.

${ }^{\mathrm{a}} p$ value for $\Delta R^{2}$. ${ }^{\mathrm{b}}$ Recalled - pre-BMT TMD.

and education to personal growth constructs that focus on perceived benefits from adversity, such as benefit finding. In several of these studies, greater personal growth was related to younger age (Curbow, Legro, et al., 1993; Klauer et al., 1998; Lechner et al., 2003; Lichtman et al., 1987; Tempelaar et al., 1989). Education was generally unrelated to measures of personal growth in these studies (Fromm et al., 1996; Katz et al., 2001; Lechner et al., 2003; Lichtman et al., 1987; Tempelaar et al., 1989; Tomich \& Helgeson, 2004), although there is one report of a relationship between higher education and greater benefit finding (Sears et al., 2003).

Findings provided partial support for hypotheses regarding relationships of appraisal and coping to posttraumatic growth. Patients who appraised BMT as producing greater emotional distress and greater concerns about survival reported greater posttraumatic growth. It is worth noting that patients' subjective concerns about mortality were associated with posttraumatic growth, whereas an objective measure of mortality risk (i.e., clinical rating of risk of disease recurrence or progression) was not. Posttraumatic growth also demonstrated expected relationships with approach coping as evidenced by significant findings for both problem solving and positive reappraisal. The latter finding is consistent with research on women with breast cancer conducted by Sears et al. (2003), in which positive reappraisal (assessed by using a different measure) was found to be significantly positively correlated with PTGI scores obtained 12 months later.

Posttraumatic growth was unrelated to concurrent reports of PTSD symptomatology. Likewise, posttraumatic growth was unrelated to pre-BMT or post-BMT levels of psychological distress. These findings are consistent with other studies of cancer patients that have found no relationship between measures of personal growth and measures of psychological distress or well-being (Cordova et al., 2001; Curbow, Somerfield, et al., 1993; Fromm et al., 1996). Additional findings from the present study based on patients' recall of pre-BMT psychological status provide some in-

\footnotetext{
${ }^{6}$ In light of this significant correlation, additional correlational analyses were conducted by using PTGI subscales. Greater perceived decline in psychological distress was significantly related to higher scores on the New Possibilities, Relating to Others, Spiritual Change, and Appreciation of Life subscales of the PTGI ( $p$ s $\leq .05)$.
} 
sight into the experience of distress over the course of BMT and its relationship to posttraumatic growth. Although there were no actual differences in distress measured before and after BMT, patients were significantly less distressed after BMT relative to their recalled status before BMT. Moreover, comparisons of actual versus recalled levels of distress before BMT suggested that the perceived decline in distress over time was attributable to overestimation (i.e., negatively biased recall) of distress prior to transplant.

Research has shown that the tendency to recall past attributes or performance in a negatively biased manner is relatively common (Wilson \& Ross, 2001). Temporal comparison theory (Albert, 1977) posits that denigrating one's past attributes is common because it facilitates a basic desire to perceive progressive improvement in oneself over time in order to maintain or enhance self-esteem. McFarland and Alvaro (2000) theorized that the tendency to engage in self-enhancing temporal comparisons may be particularly pronounced under conditions of threat and may contribute to perceptions of personal growth. Findings from the present study are consistent with this view. The amount of posttraumatic growth experienced by patients in the current study was related to perceived and not actual changes in psychological distress over time, whereby greater perceived improvement in distress was related to greater posttraumatic growth. As would be expected on the basis of temporal comparison theory, perceptions of improvement appear to be attributable to denigration of pre-BMT psychological status.

Findings from the current study should be evaluated in regard to the study's methodological limitations. First, the sample was relatively homogeneous in that a large majority of participants were White, had attended college, and were not living in poverty. Second, analyses indicated that nonparticipants were significantly younger than participants. Given that younger age was related to greater posttraumatic growth, the levels of posttraumatic growth reported may be underestimates of those present in the population. Third, order effects may have promoted biased recall of pre-BMT psychological distress. That is, obtaining retrospective ratings immediately after obtaining current ratings may have enhanced the salience of temporal comparisons. Future research should seek to examine whether biased recall is evident to the same degree if the order of administration is counterbalanced or if current and retrospective ratings are administered on different occasions.

Findings from the current study regarding the relationship of posttraumatic growth to biased recall of psychological distress should encourage additional research into the relationship of temporal comparisons to perceptions of growth. The findings reported raise the possibility that greater experience of posttraumatic growth is related to perceptions of change that are attributable to derogation of past psychological status rather than actual change in psychological status. To further investigate this issue with cancer patients, researchers should consider adapting methodology used in research on temporal comparisons in nonmedical populations (McFarland \& Alvaro, 2000; Wilson \& Ross, 2001). By substituting measures of positive personal attributes for measures of psychological distress as used in the current study, it would be possible to determine whether reports of posttraumatic growth in cancer patients reflect actual improvements over time or perceived improvements that are likely attributable to derogation of past attributes. In the absence of such research, it remains unclear whether widespread reports of personal growth following cancer diagnosis reflect a positive psychological concomitant of a stressful experience (Tedeschi et al., 1998) or an illusion motivated by the need to deal with negative thoughts and feelings engendered by a threatening event (McFarland \& Alvaro, 2000).

\section{References}

Albert, S. (1977). Temporal comparison theory. Psychological Review, 84, 485-503.

Alter, C. L., Pelcovitz, D., Axelrod, A., Goldenberg, B., Harris, H., Meyers, B., et al. (1996). Identification of PTSD in cancer survivors. Psychosomatics, 37, 137-143.

American Psychiatric Association. (1994). Diagnostic and statistical manual of mental disorders (4th ed.). Washington, DC: Author.

Andrykowski, M. A., Brady, M. J., \& Hunt, J. W. (1993). Positive psychosocial adjustment in potential bone marrow transplant recipients: Cancer as a psychosocial transition. Psycho-Oncology, 2, 261-276.

Andrykowski, M. A., Cordova, M. J., Studts, J. L., \& Miller, T. W. (1998). Posttraumatic stress disorder after treatment for breast cancer: Prevalence of diagnosis and use of the PTSD Checklist-Civilian Version (PCL-C) as a screening instrument. Journal of Consulting and Clinical Psychology, 66, 586-590.

Belec, R. H. (1992). Quality of life: Perceptions of long-term survivors of bone marrow transplantation. Oncology Nursing Forum, 19, 31-37.

Best, M., Streisand, R., Catania, L., \& Kazak, A. E. (2001). Parental distress during pediatric leukemia and posttraumatic stress symptoms (PTSS) after treatment ends. Journal of Pediatric Psychology, 26, 299307.

Brady, S. S., \& Helgeson, V. S. (1999). Social support and adjustment to recurrence of breast cancer. Journal of Psychosocial Oncology, 17(2), 37-55.

Calhoun, L. C., \& Tedeschi, R. G. (1998). Posttraumatic growth: Future directions. In R. G. Tedeschi, C. L. Park, \& L. G. Calhoun (Eds.), Posttraumatic growth: Positive changes in the aftermath of crisis (pp. 215-238). Mahwah, NJ: Erlbaum.

Carpenter, J. S., Brockopp, D. Y., \& Andrykowski, M. A. (1999). Selftransformation as a factor in the self-esteem and well-being of breast cancer survivors. Journal of Advanced Nursing, 29, 1402-1411.

Cohen, S., Mermelstein, R., Kamarck, T., \& Hoberman, H. (1985). Measuring the functional components of social support. In I. G. Sarason \& B. R. Sarason (Eds.), Social support: Theory, research, and application (pp. 73-94). The Hague, the Netherlands: Martinus Nijhoff.

Collins, R. L., Taylor, S. E., \& Skokan, L. A. (1990). A better world or a shattered vision? Changes in life perspectives following victimization. Social Cognition, 8, 263-285.

Cordova, M. J., Cunningham, L. L. C., Carlson, C. R., \& Andrykowski, M. A. (2001). Posttraumatic growth following breast cancer: A controlled comparison study. Health Psychology, 20, 176-185.

Curbow, B., Legro, M. W., Baker, F., Wingard, J. R., \& Somerfield, M. R. (1993). Loss and recovery themes of long-term survivors of bone marrow transplants. Journal of Psychosocial Oncology, 10(4), 1-20.

Curbow, B., Somerfield, M. R., Baker, F., Wingard, J. R., \& Legro, M. W. (1993). Personal changes, dispositional optimism, and psychological adjustment to bone marrow transplantation. Journal of Behavioral Medicine, 16, 423-443.

Fromm, K., Andrykowski, M. A., \& Hunt, J. (1996). Positive and negative psychosocial sequelae of bone marrow transplantation: Implications for quality of life assessment. Journal of Behavioral Medicine, 19, 221-240.

Green, B. L., Rowland, J. H., Krupnick, J. L., Epstein, S. A., Stockton, P., Stern, N. M., et al. (1998). Prevalence of posttraumatic stress disorder in women with breast cancer. Psychosomatics, 39, 102-111.

Katz, R. C., Flasher, L., Cacciapaglia, H., \& Nelson, S. (2001). The psychosocial impact of cancer and lupus: A cross validation study that 
extends the generality of "benefit finding" in patients with chronic disease. Journal of Behavioral Medicine, 24, 561-571.

Klauer, T., Ferring, D., \& Filipp, S. (1998). "Still stable after all this . . ?" Temporal comparison in coping with severe and chronic disease. International Journal of Behavioral Development, 22, 339-355.

Lechner, S. C., Zakowski, S. G., Antoni, M. H., Greenhawt, M., Block, K., \& Block, P. (2003). Do sociodemographic and disease-related variables influence benefit-finding in cancer patients? Psycho-Oncology, 12, 491499.

Lichtman, R. R., Taylor, S. E., \& Wood, J. V. (1987). Social support and marital adjustment after breast cancer. Journal of Psychosocial Oncology, 5(3), 47-74.

McFarland, C., \& Alvaro, C. (2000). The impact of motivation on temporal comparisons: Coping with traumatic events by perceiving personal growth. Journal of Personality and Social Psychology, 79, 327-343.

McNair, D. M., Lorr, M., \& Droppleman, L. F. (1992). EdITS manual for the Profile of Mood States. San Diego, CA: EdITS

Moos, R. H. (1993). Coping Responses Inventory adult form: Professional manual. Odessa, FL: Psychological Assessment Resources.

Neumann, J. K. (1991). Psychological post-traumatic effects of MI: A comparison study. Medical Psychotherapy, 4, 105-110.

Osowiecki, D. M., \& Compas, B. E. (1999). A prospective study of coping, perceived control, and psychological adaptation to breast cancer. Cognitive Therapy and Research, 23, 169-180.

Peirce, R. S., Frone, M. R., Russell, M., \& Cooper, M. (1996). Financial stress, social support, and alcohol involvement: A longitudinal test of the buffering hypothesis in a general population survey. Health Psychology, $15,38-47$.

Peltzer, K. (2000). Trauma symptom correlates of criminal victimization in an urban community sample, South Africa. Journal of Psychology in Africa; South of the Sahara, the Caribbean \& Afro-Latin America, 10, $49-62$.

Polatinsky, S., \& Esprey, Y. (2000). An assessment of gender differences in the perception of benefit resulting from the loss of a child. Journal of Traumatic Stress, 13, 709-718.

Rieker, P. P., Edbril, S. D., \& Garnick, M. B. (1985). Curative testis cancer therapy: Psychosocial sequelae. Journal of Clinical Oncology, 3, 11171126.

Schaefer, J. A., \& Moos, R. H. (1992). Life crises and personal growth. In B. N. Carpenter (Ed.), Personal coping, theory, research, and application (pp. 149-170). Westport, CT: Praeger.

Sears, S. R., Stanton, A. L., \& Danoff-Burg, S. (2003). The yellow brick road and the emerald city: Benefit finding, positive reappraisal coping, and posttraumatic growth in women with early-stage breast cancer. Health Psychology, 22, 487-497.

Shacham, S. (1983). A shortened version of the Profile of Mood States. Journal of Personality Assessment, 47, 305-306.

Snape, M. C. (1997). Reactions to a traumatic event: The good, the bad and the ugly? Psychology, Health, \& Medicine, 2, 237-242.

Tedeschi, R. G., \& Calhoun, L. G. (1995). Trauma and transformation: Growing in the aftermath of suffering. Thousand Oaks, CA: Sage.

Tedeschi, R. G., \& Calhoun, L. G. (1996). The Posttraumatic Growth Inventory: Measuring the positive legacy of trauma. Journal of Traumatic Stress, 9, 455-472.

Tedeschi, R. G., Park, C. L., \& Calhoun, L. G. (1998). Posttraumatic growth: Conceptual issues. In R. G. Tedeschi, C. L. Park, \& L. G Calhoun (Eds.), Posttraumatic growth: Positive changes in the aftermath of crisis (pp. 1-22). Mahwah, NJ: Erlbaum.

Tempelaar, R., de Haes, J. C. J. M., de Ruiter, J. H., Bakker, D., van den Heuvel, W. J. A., \& van Nieuwenhuijzen, M. G. (1989). The social experiences of cancer patients under treatment: A comparative study. Social Science and Medicine, 29, 635-642.

Tomich, P. L., \& Helgeson, V. S. (2004). Is finding something good in the bad always good? Benefit finding among women with breast cancer. Health Psychology, 23, 16-23.

Weathers, F. W., Litz, B. T., Herman, D. S., Huska, J. A., \& Keane, T. M. (1993, October). The PTSD checklist: Reliability, validity, and diagnostic utility. Paper presented at the annual meeting of the International Society for Traumatic Stress Studies, San Antonio, TX.

Weiss, T. (2002). Posttraumatic growth in women with breast cancer and their husbands: An intersubjective validation study. Journal of Psychosocial Oncology, 20(2), 65-80.

Widows, M. R., Jacobsen, P. B., \& Fields, K. K. (2000). Relation of psychological vulnerability factors to posttraumatic stress disorder symptomatology in bone marrow transplant recipients. Psychosomatic Medicine, 62, 873-882.

Wilson, A. E., \& Ross, M. (2001). From chump to champ: People's appraisals of their earlier and present selves. Journal of Personality and Social Psychology, 80, 572-584.

Zemore, R., Rinholm, J., Shepel, L. F., \& Richards, M. (1989). Some social and emotional consequences of breast cancer and mastectomy: A content analysis of 87 interviews. Journal of Psychosocial Oncology, 7(4), $33-45$.

Zemore, R., \& Shepel, L. F. (1989). Effects of breast cancer and mastectomy on emotional support and adjustment. Social Science and Medicine, 28, 19-27.

\section{E-Mail Notification of Your Latest Issue Online!}

Would you like to know when the next issue of your favorite APA journal will be available online? This service is now available to you. Sign up at http://watson.apa.org/ notify/ and you will be notified by e-mail when issues of interest to you become available! 